\title{
Contribution à l'étude des variations de la teneur en sels minéraux du lait de vache dans différentes régions françaises
}

\author{
par \\ L. MOUILLET, F. M. LUQUET et J. CASALIS \\ Laboratoire d'Industrie Laitière de Douai
}

\section{INTRODUCTION}

La composition minérale du lait de vache a fait l'objet de nombreuses publications et depuis l'application de la spectrométrie d'absorption atomique, l'on connaît d'une façon assez précise les teneurs moyennes des différents éléments minéraux du lait. Des travaux ont été effectués à différentes reprises afin de rechercher les causes des variations de ces teneurs. Les différents facteurs mis en évidence par des chercheurs tels que Gueguen et Morre $[6,12]$, Smith [20], Miller [12], Kamal [8] et Felder [3], sont la race, la santé, le stade de lactation, la productivité, l'alimentation et l'environnement (sols, climat, etc.) de l'animal.

Une étude publiée récemment [20] nous avait permis de constater d'une part, qu'il y avait entre les laits alimentant le même jour deux usines d'une même firme laitière situées l'une dans le Nord et l'autre dans le Sud-Ouest de la France des différences notables particulièrement en ce qui concerne les taux de potassium, de calcium et de phosphore, d'autre part que ces différences avaient des répercussions sur le processus d'acidification et sur la texture du caillé des laits fermentés et des fromages fabriqués par ces deux usines. Dans le cadre d'un travail de fin d'études d'élève ingénieur de l'Institut Supérieur d'Agriculture nous avons élargi ces travaux en effectuant sur le plan national une étude statistique, sans avoir pour autant la possibilité d'étudier en détail les causes des variations constatées.

\section{I. - CONDITIONS EXPERIMENTALES}

\section{$1^{\circ}$ Echantillonnage}

Pour découper la France en régions agricoles nous avons tenu compte à la fois de critères géographiques et de la répartition des races bovines (tab. 1). 
TABLEAU 1

Principales races bovines et effectifs du troupeau laitier français en milliers de têtes (1972)

\begin{tabular}{|c|c|c|c|c|c|c|}
\hline Régions & FFPN & Normande & $\begin{array}{l}\text { Pie Rouge } \\
\text { de l'Est }\end{array}$ & Charolaise & Limousine & Maine - Anjou \\
\hline & & & & 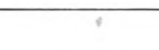 & & \\
\hline Nord & 515,9 & 27,8 & - & 13,4 & 0,1 & 1,0 \\
\hline Normandie & 91,1 & 918,2 & - & 3,8 & - & 0,3 \\
\hline Bretagne & 211,7 & 632,6 & - & 1,6 & - & 3,7 \\
\hline Pays de la Loire - Centre & 303,6 & 640,3 & 0,6 & 175,5 & 13,7 & . 137 \\
\hline Charentes - Poitou & 215,5 & 163 & - & 42,2 & 20 & 9 \\
\hline Massif Central & 117,5 & 47,9 & 98,1 & 228,2 & 219,5 & - \\
\hline Sud-Ouest - Languedoc & 521,6 & 82,8 & 22,7 & 14,9 & 57,8 & - \\
\hline Provence - Rhône-Alpes - Sud-Est & 101 & 0,3 & 383,6 & 35,4 & - & - \\
\hline Jura - Bourgogne & 66,6 & 28,6 & 380 & 246,1 & - & - \\
\hline Est & 553,7 & 2,3 & 166 & 24,2 & 0,3 & - \\
\hline
\end{tabular}


En définitive et en soulignant le caractère assez arbitraire de ce choix nous avons retenu les dix régions suivantes (fig. 1).
A - Nord.
F - Massif Central.
B - Normandie.
G - Sud-Ouest - Pyrénées.
C - Bretagne.
$\mathrm{H}$ - Alpes.
D - Pays de Loire.
I - Bourgogne - Jura.
E - Charentes-Poitou.
$\mathrm{J}-$ Est.

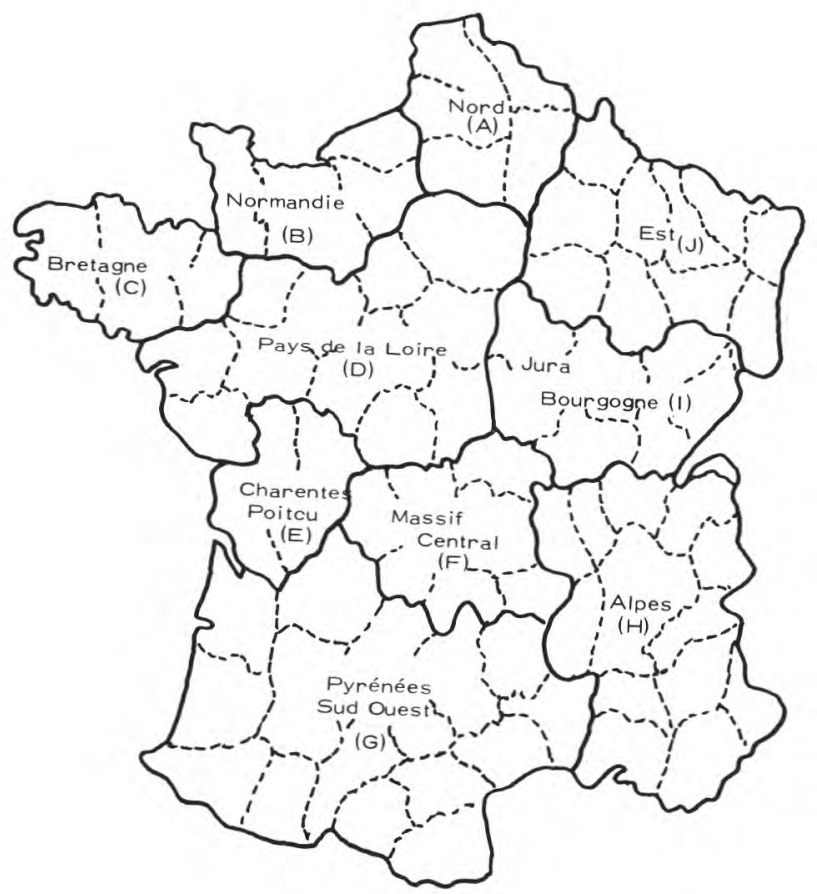

fig 1

Au sein de chacune de ces régions, nous avons obtenu le concours de dix usines importantes : des échantillons de lait entier de grand mélange, représentatifs de l'ensemble des tournées de ramassage de chacune d'entre elles ont été prélevés deux fois par mois à date fixe d'avril à juillet 1974. Ces échantillons additionnés d'aldéhyde formique à $30 \mathrm{p} .100$ à raison de $0,1 \mathrm{ml}$ pour $250 \mathrm{ml}$ de lait étaient envoyés au Laboratoire d'Industrie Laitière de Douai avec l'indication du litrage collecté par l'usine le jour du prélèvement. Ainsi les analyses ont porté sur 800 échantillons représentant au total une quantité de lait d'environ 2 millions d'hl. 


\section{$2^{\circ}$ Analyses}

Les déterminations suivantes ont été effectuées sur chacun des échantillons :

- Macro-éléments : calcium, magnésium, potassium, sodium.

- Oligo-éléments : cuivre, fer, manganèse, zinc, par spectrométrie d'absorption atomique, en utilisant au préalable, soit une défécation au moyen d'une solution d'acide trichloracétique pour le dosage du calcium, du magnésium, du potassium et du sodium [4] soit une minéralisation par voie humide à l'aide d'un mélange sulfonitrique et du dispositif proposé par Pien [16] pour le fer, ou par voie sèche à $500^{\circ} \mathrm{C}$ pour le cuivre, le manganèse et le zinc [22].

\section{II. - RESULtATS}

A partir des différentes séries de résultats sont calculés les moyennes arithmétiques régionales $\overrightarrow{\mathrm{x}}$ et nationales $\overrightarrow{\mathrm{x}}$ et leurs écartstypes appelés respectivement $\sigma_{1}$ et $\sigma_{2}$ (tab. 2, 3). Il nous a paru intéressant d'inclure dans le tableau 3, à titre de comparaison, les teneurs indiquées dans une récente publication de Gueguen [6].

\section{III. - DISCUSSION}

\section{$1^{\circ}$ Signification des résultats}

La faible valeur des écarts-types concernant le calcium, le magnésium, le manganèse, le potassium et le sodium permet de penser que la moyenne obtenue pour chacun de ces éléments est significative. Par contre, les dispersions des valeurs autour de la moyenne, constatées au cours de cette étude surtout pour des éléments tels que le cuivre, le fer ou le zinc, sont assez importantes et du même ordre de grandeur que celles relevées dans d'autres travaux [6, 22]. L'unanimité n'est pas encore faite en ce qui concerne les teneurs moyennes du lait en ces éléments.

\section{$2^{\circ}$ Facteurs de variations}

Des études citées précédemment ont déjà été consacrées à ce problème et, étant donné qu'il a été retenu, pour le découpage de la France en régions agricoles, le critère géographique et celui des races laitières, il est possible de commenter quelques-uns des résultats obtenus en fonction de ces paramètres. 
TABLEAU 2. - Teneurs régionales moyennes et écarts-types des éléments minéraux du lait

\begin{tabular}{|c|c|c|c|c|c|c|c|c|c|}
\hline Régions & $\begin{array}{l}\text { Eléments } \\
\text { minéraux }\end{array}$ & $\underset{\mathrm{g} / 1}{\mathrm{~K}}$ & $\begin{array}{l}\mathrm{Ca} \\
\mathrm{g} / \mathrm{l}\end{array}$ & $\begin{array}{l}\mathrm{Na} \\
\mathrm{g} / \mathrm{l}\end{array}$ & $\begin{array}{l}\mathrm{Mg} \\
\mathrm{g} / 1\end{array}$ & $\underset{\mathrm{mg} / \mathrm{Zn}}{\mathrm{Zn}}$ & $\begin{array}{c}\mathrm{Fe} \\
\mu \mathrm{g} / \mathrm{l}\end{array}$ & $\underset{\mu \mathrm{g} / \mathrm{l}}{\mathrm{Cu}}$ & $\begin{array}{c}\mathrm{Mn} \\
\mu \mathrm{g} / \mathrm{l}\end{array}$ \\
\hline Alpes & $\begin{array}{l}\overline{\mathrm{x}} \\
\sigma_{1}\end{array}$ & $\begin{array}{l}1,525 \\
0,019\end{array}$ & $\begin{array}{l}1,228 \\
0,061\end{array}$ & $\begin{array}{l}0,446 \\
0,027\end{array}$ & $\begin{array}{l}0,112 \\
0,005\end{array}$ & $\begin{array}{l}4,07 \\
0,29\end{array}$ & $\begin{array}{r}433,0 \\
54,0\end{array}$ & $\begin{array}{r}109,5 \\
15,1\end{array}$ & $\begin{array}{r}57,7 \\
7,1\end{array}$ \\
\hline Bretagne & $\begin{array}{l}\bar{x} \\
\sigma_{1}\end{array}$ & $\begin{array}{l}1,454 \\
0,041\end{array}$ & $\begin{array}{l}1,265 \\
0,026\end{array}$ & $\begin{array}{l}0,497 \\
0,034\end{array}$ & $\begin{array}{l}0,120 \\
0,007\end{array}$ & $\begin{array}{l}4,58 \\
0,29\end{array}$ & $\begin{array}{r}322,0 \\
63,0\end{array}$ & $\begin{array}{r}100,0 \\
15,5\end{array}$ & $\begin{array}{r}59,4 \\
8,4\end{array}$ \\
\hline Charentes - Poitou & $\begin{array}{l}\bar{x} \\
\sigma_{1}\end{array}$ & $\begin{array}{l}1,533 \\
0,016\end{array}$ & $\begin{array}{l}1,260 \\
0,042\end{array}$ & $\begin{array}{l}0,493 \\
0,022\end{array}$ & $\begin{array}{l}0,107 \\
0,005\end{array}$ & $\begin{array}{l}5,57 \\
0,29\end{array}$ & $\begin{array}{r}541,0 \\
47,0\end{array}$ & $\begin{array}{r}110,0 \\
24,1\end{array}$ & $\begin{array}{r}42,4 \\
5,2\end{array}$ \\
\hline Est & $\begin{array}{l}\bar{x} \\
\sigma_{1}\end{array}$ & $\begin{array}{l}1,557 \\
0,22\end{array}$ & $\begin{array}{l}1,180 \\
0,046\end{array}$ & $\begin{array}{l}0,427 \\
0,020\end{array}$ & $\begin{array}{l}0,109 \\
0,004\end{array}$ & $\begin{array}{l}4,14 \\
0,17\end{array}$ & $\begin{array}{r}372,0 \\
54,0\end{array}$ & $\begin{array}{r}126,0 \\
41,9\end{array}$ & $\begin{array}{r}50,0 \\
7,4\end{array}$ \\
\hline Jura - Bourgogne & $\begin{array}{l}\overline{\mathrm{x}} \\
\sigma_{1}\end{array}$ & $\begin{array}{l}1,497 \\
0,028\end{array}$ & $\begin{array}{l}1,252 \\
0,037\end{array}$ & $\begin{array}{l}0,397 \\
0,019\end{array}$ & $\begin{array}{l}0,104 \\
0,006\end{array}$ & $\begin{array}{l}3,91 \\
0,38\end{array}$ & $\begin{array}{r}371,0 \\
60,0\end{array}$ & $\begin{array}{r}156,0 \\
41,5\end{array}$ & $\begin{array}{r}52,2 \\
8,4\end{array}$ \\
\hline Massif Central & $\begin{array}{l}\overline{\mathrm{x}} \\
\sigma_{1}\end{array}$ & $\begin{array}{l}1,454 \\
0,038\end{array}$ & $\begin{array}{l}1,188 \\
0,048\end{array}$ & $\begin{array}{l}0,438 \\
0,024\end{array}$ & $\begin{array}{l}0,112 \\
0,006\end{array}$ & $\begin{array}{l}4,22 \\
0,26\end{array}$ & $\begin{array}{r}462,0 \\
75,0\end{array}$ & $\begin{array}{r}109,0 \\
21,1\end{array}$ & $\begin{array}{r}56,8 \\
8,2\end{array}$ \\
\hline Nord & $\begin{array}{l}\overline{\mathrm{x}} \\
\sigma_{1}\end{array}$ & $\begin{array}{l}1,546 \\
0,053\end{array}$ & $\begin{array}{l}1,207 \\
0,027\end{array}$ & $\begin{array}{l}0,448 \\
0,025\end{array}$ & $\begin{array}{l}0,111 \\
0,003\end{array}$ & $\begin{array}{l}4,61 \\
0,28\end{array}$ & $\begin{array}{r}371,0 \\
83,0\end{array}$ & $\begin{array}{r}141,5 \\
40,0\end{array}$ & $\begin{array}{r}60,0 \\
7,8\end{array}$ \\
\hline Normandie & $\begin{array}{l}\overline{\mathrm{x}} \\
\sigma_{1}\end{array}$ & $\begin{array}{l}1,387 \\
0,056\end{array}$ & $\begin{array}{l}1,249 \\
0,050\end{array}$ & $\begin{array}{l}0,424 \\
0,027\end{array}$ & $\begin{array}{l}0,113 \\
0,005\end{array}$ & $\begin{array}{l}4,71 \\
0,45\end{array}$ & $\begin{array}{r}512,0 \\
78,0\end{array}$ & $\begin{array}{r}122,0 \\
24,1\end{array}$ & $\begin{array}{r}63,6 \\
8,6\end{array}$ \\
\hline Pays de la Loire & $\begin{array}{l}\bar{x} \\
\sigma_{1}\end{array}$ & $\begin{array}{l}1,410 \\
0,028\end{array}$ & $\begin{array}{l}1,210 \\
0,049\end{array}$ & $\begin{array}{l}0,443 \\
0,032\end{array}$ & $\begin{array}{l}0,107 \\
0,005\end{array}$ & $\begin{array}{l}4,29 \\
0,40\end{array}$ & $\begin{array}{r}381,0 \\
80,0\end{array}$ & $\begin{array}{r}148,0 \\
48,3\end{array}$ & $\begin{array}{r}56,6 \\
9,6\end{array}$ \\
\hline Pyrénées - Sud-Ouest & $\begin{array}{l}\bar{x} \\
\sigma_{1}\end{array}$ & $\begin{array}{l}1,547 \\
0,024\end{array}$ & $\begin{array}{l}1,201 \\
0,051\end{array}$ & $\begin{array}{l}0,449 \\
0,023\end{array}$ & $\begin{array}{l}0,109 \\
0,004\end{array}$ & $\begin{array}{l}3,97 \\
0,14\end{array}$ & $\begin{array}{r}458,0 \\
84,0\end{array}$ & $\begin{array}{r}145,5 \\
45,9\end{array}$ & $\begin{array}{r}56,6 \\
8,7\end{array}$ \\
\hline
\end{tabular}


TABLEAU 3. - Teneurs moyennes nationales et écarts-types des éléments minéraux du lait

\begin{tabular}{|c|c|c|c|c|c|c|c|c|c|}
\hline \multicolumn{2}{|l|}{ Eléments minéraux } & $\begin{array}{c}\mathrm{K} \\
\mathrm{g} / \mathrm{l}\end{array}$ & $\begin{array}{l}\mathrm{Ca} \\
\mathrm{g} / \mathrm{l}\end{array}$ & $\begin{array}{l}\mathrm{Na} \\
\mathrm{g} / \mathrm{l}\end{array}$ & $\begin{array}{l}\mathrm{Mg} \\
\mathrm{g} / 1\end{array}$ & $\underset{\mathrm{mg} / \mathrm{Z}}{\mathrm{Zn}}$ & $\underset{\mu \mathrm{g} / 1}{\mathrm{Fe}}$ & $\underset{\mu \mathrm{g} / 1}{\mathrm{Cu}}$ & $\underset{\mu \mathrm{g} / \mathrm{l}}{\mathrm{Mn}}$ \\
\hline Moyenne Nationale & $\overline{\mathrm{x}}$ & 1,488 & 1,225 & 0,445 & 0,110 & 4,41 & 422,0 & 126,5 & 55,5 \\
\hline Ecart-type & $\sigma_{2}$ & 0,059 & 0,030 & 0,030 & 0,004 & 0,49 & 70,0 & 19,8 & 6,0 \\
\hline Travaux de Gueguen & & 1,500 & 1,250 & 0,520 & 0,120 & $2,0-5,0$ & $200-500$ & $20-150$ & $30-50$ \\
\hline
\end{tabular}


a) INFLUENCE DE LA RACE

a.1. Macro-éléments : La plus grande partie du potassium et du sodium est en solution dans la phase liquide du lait, tandis que le calcium et le magnésium sont principalement en combinaison avec les protéines. Les laits à forte teneur protéique sont plus riches en calcium et en magnésium, plus pauvres en potassium et en sodium que les laits à faible teneur protéique [6]. La teneur du lait en protéines étant différente suivant les races laitières, il existe donc une relation entre race des animaux et teneur du lait en macro-éléments [11]. Ces indications se confirment en examinant les résultats analytiques figurant au tableau 2 à propos des teneurs en calcium, potassium et sodium des échantillons de lait provenant de Normandie, du Nord, des Pyrénées, des Alpes et de l'Est en tenant compte de la répartition géographique des races laitières (cf. tab. 1) : le lait des vaches normandes est plus riche en calcium mais plus pauvre en potassium et en sodium que le lait des races FFPN et Pie Rouge de l'Est.

a.2. Oligo-éléments : Différents auteurs ont mis en évidence l'influence de facteurs d'ordre génétique sur la composition en oligoéléments du lait $[1,6]$. Le fer, le cuivre et le zinc étant adsorbés à la surface des globules gras, il est possible, comme l'a écrit Morre [13], qu'il y ait une relation entre la teneur du lait en ces oligoéléments et sa richesse en matière grasse. Prenons par exemple la teneur en zinc, des laits du Massif Central, des Pyrénées, des Alpes, du Jura et de l'Est, qui est plus faible que celle des autres régions : l'influence des races peut expliquer cette différence : d'un côté dominent les races Pie Rouge de 1'Est, Brune des Alpes, Charolaise et Limousine ; de l'autre se rencontrent plus fréquemment les races normandes et FFPN.

En définitive nous avons rassemblé quelques éléments d'appréciation dans le tableau 1, qui montre une certaine corrélation entre des races telles que FFPN, Normande ou Pie Rouge de l'Est et la composition minérale du lait.

\section{b) INFLUENCE DE LA PRODUCTION LAITIÈRE}

Bien que Kirchgessner [9] ait constaté qu'avec de fortes productions laitières, la teneur en sodium diminuait, ce facteur ne semble pas avoir d'influence sur les macro-éléments : ainsi la région Nord et celle du Sud-Ouest, ayant approximativement la même répartition de races dans leur cheptel bovin, mais des productivités différentes (tabl. 1, 4), ont des teneurs très voisines en calcium, magnésium, potassium et sodium. En ce qui concerne les oligo-éléments, il semble difficile, là encore d'incriminer ce facteur, car dans certains cas, il paraît intervenir un phénomène de dilution (Est - Sud-Ouest), mais, 
TABLEAU 4. - Productivité laitière en litres de lait par animal (1972) (d'après l'I.T.E.B.)

\begin{tabular}{|c|c|c|c|c|c|c|c|}
\hline Régions & FFPN & Normande & $\begin{array}{l}\text { Pie Rouge } \\
\text { de l'Est }\end{array}$ & $\begin{array}{l}\text { Maine } \\
\text { Anjou }\end{array}$ & Flamande & $\begin{array}{l}\text { Bretonne } \\
\text { Pie Noire }\end{array}$ & $\begin{array}{l}\text { Brune des } \\
\text { Alpes }\end{array}$ \\
\hline Nord & 3600 & 3415 & - & - & 2610 & - & - \\
\hline Normandie & 3610 & 3250 & - & 3100 & 3500 & - & - \\
\hline Bretagne & 3420 & 2790 & 2640 & 2300 & - & 2140 & - \\
\hline Pays de la Loire - Centre & 3420 & 2940 & 2760 & 2510 & 2740 & - & - \\
\hline Charentes - Poitou & 3250 & 2860 & - & 2360 & 3420 & - & - \\
\hline Massif Central & 2825 & 2860 & 2630 & - & - & - & 2390 \\
\hline Sud-Ouest - Languedoc & 2680 & 2450 & 2315 & - & - & 2570 & 2100 \\
\hline Sud-Est & 3040 & - & 2955 & - & - & 3000 & 3070 \\
\hline Jura - Bourgogne & 3270 & 2920 & 3300 & - & - & - & 3100 \\
\hline Est & 3185 & 2885 & 2930 & - & - & - & 2810 \\
\hline
\end{tabular}


TABLEAU 5. - Races laitières et composition minérale du lait

\begin{tabular}{|c|c|c|c|c|c|c|c|c|c|}
\hline $\begin{array}{l}\text { Races } \\
\text { dominantes }\end{array}$ & $\begin{array}{l}\text { Code } \\
\text { des } \\
\text { régions }\end{array}$ & $\mathrm{K}$ & $\mathrm{Ca}$ & $\mathrm{Na}$ & $\mathrm{Mg}$ & $\mathrm{Zn}$ & $\mathrm{Fe}$ & $\mathrm{Cu}$ & Mn \\
\hline FFPN & $\begin{array}{l}\mathrm{A} \\
\mathrm{G} \\
\mathrm{J}\end{array}$ & $\begin{array}{l}+ \\
+ \\
+\end{array}$ & $\begin{array}{l}- \\
\overline{-}\end{array}$ & $\begin{array}{l}+ \\
+ \\
+\end{array}$ & $\begin{array}{l}\mathrm{O} \\
\mathrm{O} \\
\mathrm{O}\end{array}$ & $\frac{+}{-}$ & $\begin{array}{l}- \\
+\end{array}$ & $\begin{array}{l}+ \\
+ \\
+\end{array}$ & $\stackrel{+}{0}$ \\
\hline Normande & $\begin{array}{l}\text { B } \\
\text { C }\end{array}$ & - & $\begin{array}{l}+ \\
+\end{array}$ & $\overline{+}$ & $\begin{array}{l}+ \\
+\end{array}$ & $\begin{array}{l}+ \\
+\end{array}$ & $\stackrel{++}{--}$ & -- & $\begin{array}{l}+ \\
+\end{array}$ \\
\hline Pie Rouge de l'Est & $\stackrel{\mathrm{H}}{\mathrm{I}}$ & $\begin{array}{l}+ \\
+\end{array}$ & $\begin{array}{l}+ \\
+\end{array}$ & $\frac{0}{-}$ & \pm & $\overline{-}$ & \pm & $\overline{++}$ & \pm \\
\hline FFPN - Normande & $\mathrm{E}$ & + & + & ++ & - & ++ & ++ & - & - \\
\hline Limousine - Charolaise & $\mathrm{F}$ & - & - & o & $\mathrm{O}$ & - & ++ & - & + \\
\hline $\begin{array}{l}\text { FFPN - Normande } \\
\text { Charolaise - Maine - Anjou }\end{array}$ & $\mathrm{D}$ & - & - & $\mathrm{O}$ & - & - & - & + & $\mathrm{O}$ \\
\hline $\begin{array}{ll}- & \text { inférieur à la moyenne } \\
- \text { : } & \text { très inférieur à la moy } \\
\mathrm{O}: & \text { sensiblement égal à la }\end{array}$ & $\begin{array}{l}\text { ionale. } \\
\text { enationa } \\
\text { enne na }\end{array}$ & & & $\begin{array}{r}+: \\
++: \\
\square: \\
\square\end{array}$ & $\begin{array}{l}\text { Ir à } \\
\text { érie } \\
\text { s mi }\end{array}$ & $\begin{array}{l}\text { enn } \\
\text { moy } \\
\text { sus }\end{array}$ & $\begin{array}{l}\text { ionale. } \\
\text { nation } \\
\text { bles d'ê }\end{array}$ & h re & vec une \\
\hline
\end{tabular}


on peut observer un effet contraire dans les laits en provenance de Normandie.

\section{c) Facteurs climatiques et géographiques}

Différents auteurs [8] ont pu mettre en évidence l'influence de la saison sur la composition minérale du lait. Mais nous ne prendrons pas ce facteur en considération, étant donné que les échantillons ont été prélevés dans chacune des régions durant la même période de l'année.

Par contre les chiffres du tableau 2, semblent confirmer les travaux de Kamal [8] d'après lesquels les teneurs en calcium et potassium varient en sens inverse de la température. En effet, ces éléments sont présents en plus faibles quantités dans les laits de la région Pyrénées - Sud-Ouest que dans ceux du Nord de la France ; ces différences pourraient être dues à une consommation plus faible d'aliments secs, due à une température plus élevée dans la région Pyrénées - Sud-Ouest que dans celle du Nord de la France [20], d'où une prise de minéraux plus réduite [8].

Le sol a une influence, souvent indirecte, en relation avec l'alimentation et le climat. Une étude de Felber [3] dans les Pré-Alpes du Nord (calcaires) et les Alpes Centrales (roches granitiques) laisse apparaître le rôle du sol en tant que fournisseur de minéraux. Toutefois dans le cadre de nos travaux ce facteur paraît difficile à mettre en évidence, étant donné la grande diversité des sols à l'intérieur même de chaque région.

\section{CONCLUSION}

Cette étude, bien que limitée, a permis d'une part de rassembler des données analytiques sur la composition minérale des laits de vache en France : ces résultats font ressortir les différences pouvant exister d'une région à l'autre et montrent que ces différences peuvent être dues, en partie, à des facteurs génétiques, surtout mis en évidence, pour les races FFPN, Normande et Pie Rouge de l'Est. II serait possible de donner à un tel type d'enquête d'autres orientations, soit en délimitant un plus grand nombre de régions (mais dans cette hypothèse la quantité d'échantillons à analyser risquerait de dépasser les capacités analytiques d'un seul laboratoire), soit en étudiant des variations localisées, à l'intérieur d'une région déterminée et qui seraient exploitables par une ou plusieurs usines situées dans cette région. Dans les deux cas il serait possible alors d'étudier plus en détail les causes des variations de la teneur en sels minéraux, de mieux expliquer leur influence sur le déroulement de certains processus technologiques, et éventuellement de remédier aux anomalies constatées dans ce déroulement. 


\section{Rés u mé}

A l'intérieur de dix régions françaises, sélectionnées en fonction des critères géographiques et de la répartition des races bovines, il a été prélevé deux fois par mois, d'avril à juillet 1974, des échantillons de laits de grand mélange, représentatifs de l'ensemble des tournées de ramassage des usines les plus importantes.

Sur chacun des échantillons ont été effectuées, par spectrométrie d'absorption atomique, les déterminations suivantes : calcium, magnésium, potassium, sodium, cuivre, fer, manganèse et zinc.

Une étude statistique a été réalisée sur chacune des séries de résultats afin d'obtenir des moyennes régionales et nationales ainsi que les écarts-types correspondants.

L'influence de la race bovine sur la composition en sels minéraux du lait a pu être mise en évidence surtout en ce qui concerne les races FFPN, Normande et Pie Rouge de l'Est.

\section{S u m m a r y}

Within ten french regions, selected by their differences on geographical conditions and cattle breeds, bulk-milk samples, coming from most important dairies, have been collected twice a month, during four months, from April to July 1974.

Each sample was assayed for calcium, magnesium, potassium, sodium, copper, iron, manganese and zinc by atomic absorption spectrometry.

Regional and national averages and their standard deviation has been evaluated. The mineral content of the milk depends for a large part upon the cow's breed, especially in case of FFPN, Normande and Pie Rouge de l'Est.

Reçu pour publication en juillet 1975.

\section{Bibliographie}

[1] Auriol (P.), Mocouot (G.) (1962). - Quelques facteurs de variation de la teneur en calcium des laits de vache. J. Dairy Res., 29, 189-191.

[2] Bouzoun (I.) (1966). - Teneur en potassium, sodium, magnésium dans le lait lorsque les vaches sont nourries avec des aliments ensilés et des tourteaux. Tech. Lait., (521), 17-25.

[3] Felber (H.) (1970). - Zur labgerinnung der silage. Milch - teil II : Mineral und eiweissgehalt von silage und nicht - silage - milch. Die osterreichische milchwirtschaft, 25, (2), 1-11. 
[4] Frazeur (D. R.) (1967). - Determination of selected cations in milk by atomic absorption spectrophotometry. J. Dairy Sci., 50, 938.

[5] GaUnT (S. N.) (1972). - Genetic and environmental changes possible in milk composition. J. Dairy Sci., 56, (2), 270-278.

[6] Gueguen (L.) (1971). - La composition minérale du lait et son adaptation aux besoins minéraux du jeune. Ann. Nutrit. Aliment., 25, A 335 -A 381.

[7] Gueguen (L.), Journet (M.) (1961). - Les variations de la composition minérale du lait de vache. Ann. Biol. anim. Biochim. Biophys., 305-310.

[8] Kamal (T. H.), Johnson (H. D.) and Ragsdale (A. C.) (1961). - Influence of the stade of lactation and environmental temperatures on the salt balance of milk. J. Dairy Sci., 44, 1655.

[9] Kirchgessner (M.), Friesecke (H.), Koch (G.) (1967). - Nutrition and the composition of milk. Ed. Crosby Lockwood and Son Ltd. London.

[10] Linden (G.) (1971). - Applications de la spectrophotométrie d'absorption atomique dans les laboratoires d'industrie laitière. Industr. aliment. agric., 6, 793-803.

[11] Madelmont (C.), Michon (G.) (1965). - Teneur en potassium des laits de provenances diverses. Bull. Acad. Vétér. Fr., tome XXXVIII.

[12] Miller (M.) (1970). - Zinc nutrition of cattle. J. Dairy Sci., 53, (8), 1123-1132.

[13] Morre (J.) (1974). - Les métaux, agents de pollution du lait. Leur dosage par spectrophotométrie d'absorption atomique. Le Lait, (533-534), 139-152.

[14] Nickerson (T. A.) (1959). - Chemical composition of milk. Food Technol., 128, 598-606.

[15] Pien (J.) (1956). - Le dosage des métaux et des métalloïdes dans les produits laitiers. XIVe Congrès International de Laiterie, Rome, sept., vol. 3 , partie 2, section 3, 398-405.

[16] Pinta (M.) (1962). - Recherche et dosage des éléments traces. Ed Dunod, Paris.

[17] Pinta (M.) (1971). - Spectrométrie d'absorption atomique. Ed. Masson, Paris.

[18] RathoRe (A. K.) (1970). - Influence of age on milk and milk constituents and their relationships in lactating cows. Austral. J. Dairy Technol., 19, 106-108.

[19] Rook (J.A.), Wood (M.) (1959). - Potassium and lactose in milk in relation to the phisiology of milk secretion. Nature, 184,647 .

[20] Roux (M.), Luouet (F. M.), Casalis (J.) (1974). - Sur la teneur en sels minéraux de laits de vache des régions du Nord et du Sud-Ouest de la France. Ann. Falsi. Exp. Chim., nov,-déc., 67, (723-724), 577-583.

[21] Sasser (L.B.), Ward (G. M.), Johnson (J. E.) (1966). - Variations in potassium concentration of cow's milk. J. Dairy Sci., 49, (12), 893.

[22] Sмith (A.C.). - Rapid methods for determining copper content of milk. J. Dairy Sci., 50, (5), 664-668. 